\title{
BMJ Open Perceived barriers to multiprofessional team briefings in operating theatres: a qualitative study
}

\author{
Laura Fruhen (D) , ${ }^{1}$ Joseph Alexandre Carpini (D) , ${ }^{2}$ Sharon K Parker, ${ }^{3}$ Yee Leung, ${ }^{4}$ \\ Adrian F S Flemming ${ }^{5}$
}

To cite: Fruhen $L$, Carpini JA, Parker SK, et al. Perceived barriers to multiprofessional team briefings in operating theatres: a qualitative study. BMJ Open 2020;10:e032351. doi:10.1136/ bmjopen-2019-032351

- Prepublication history and additional material for this paper are available online. To view these files, please visit the journal online (http://dx.doi. org/10.1136/bmjopen-2019032351).

Received 17 June 2019

Revised 22 November 2019

Accepted 30 December 2019

Check for updates

(c) Author(s) (or their employer(s)) 2020. Re-use permitted under CC BY-NC. No commercial re-use. See rights and permissions. Published by BMJ.

For numbered affiliations see end of article.

Correspondence to

Dr Laura Fruhen;

laura.fruhen@uwa.edu.au

\section{ABSTRACT}

Objectives This study investigates perceived barriers towards the implementation of multiprofessional team briefings (MPTB) in operating theatres, as well as ways to overcome these perceived barriers. Previous research shows that MPTB can enhance teamwork and communication, but are underused in operating theatres. By adopting a multilevel systems perspective, this study examines perceived barriers and solutions for MPTB implementation.

Design Participants completed open-ended survey questions. Responses were coded via qualitative content analysis. The analysis focused on themes in the responses and the systems level at which each barrier and solution operates.

Setting Four tertiary hospitals in Australia.

Participants 103 operating theatre staff, including nurses, surgeons, anaesthetists, technicians and administrators.

Results Participants identified barriers and solutions at the organisational (15.81\% of barriers; $74.10 \%$ of solutions), work group (61.39\% of barriers; $25.09 \%$ of solutions) and individual level $(22.33 \%$ of barriers; $0 \%$ of solutions). Of all the perceived barriers to MPTB occurrence, a key one is getting everyone into the room at the same time. Matching of perceived barriers and solutions shows that higher systems-level solutions can address lower level barriers, thereby showing the relevance of implementing such wider reaching solutions to MPTB occurrence (including work practices at occupational level and above) as well as addressing more local issues.

Conclusions Successful MPTB implementation requires changes at various systems levels. Practitioners can strategically prepare and plan for systems-based strategies to overcome barriers to MPTB implementation. Future research can build on this study's findings by directly examining higher systems-level barriers and solutions via detailed case analyses.

\section{BACKGROUND}

The purpose of this study is to identify the perceived barriers and solutions towards the successful implementation of multiprofessional team briefings (MPTB) in operating theatres (OTs). Preoperative communication between staff is not well studied, yet is reported as underused and lacking a standard method

\section{Strengths and limitations of this study}

This study investigates barriers and solutions to the implementation of multiprofessional team briefings (MPTB), as perceived by operating theatre staff $(n=103)$.

- Open-ended questions allowed participants to freely bring up topics that were salient to them.

- Inductive analysis of participants' responses adopted a multilevel systems model to identify barriers and solutions to MPTB implementation.

- The sample was of sufficient size and represented key stakeholders in operating theatres.

- This study is only descriptive and there may be differences between perceived and actual barriers to MPTB implementation.

or procedure. ${ }^{1}$ MPTB serve as a potential standardised complement to surgical checklists that can enhance preoperative communication and hence theatre performance and safety. ${ }^{2}$ In OTs across the globe, surgical checklists have been widely adopted. The introduction of checklists has had clear benefits to patient outcomes in terms of improved detection of safety hazards, reduced postoperative mortality and reduced complication rates. ${ }^{34}$ Nonetheless, there is still room for further improvement in how surgical teams work together. Globally, $16.8 \%$ of patients undergoing elective surgery develop one or more postoperative complication and $0.5 \%$ die. ${ }^{5}$ The fact that preventable complications still occur in OTs despite the implementation of surgical checklists suggests that surgical checklists alone cannot fully address the dynamic issues that contribute to negative patient and surgical team outcomes. ${ }^{16}$

Preoperative communication and MPTB specifically have been identified as a complementary approach to checklists that provides opportunity for team building and addresses sociocultural aspects related to teamwork that checklists do not directly address. Importantly, 
MPTB offer the opportunity for teams to develop shared mental models, effective team work behaviours and communication. ${ }^{2}$ MPTB are short meetings, conducted at the beginning of a surgical list before the first patient arrives. ${ }^{7}$ This timing has been rated favourably by surgical staff, compared with the timing of the checklists immediately prior to the start of each procedure. ${ }^{1}$ They include those surgeons, nurses, anaesthetists and technicians involved in an OT list. The purpose of MPTB is to enable theatre staff to share information, to create a team identity where information can be freely exchanged and to plan ahead across the full list. In this way, MPTB are distinct from and complement checklists and may address some of the underlying issues still contributing to preventable surgical complications and never events. ${ }^{8}$ MPTB at the start of operating lists vary with regard to their structure and content. Leong $e t a l^{7}$ followed three steps (introduction round, tasks of the team members and expected technical or logistical issues that require extra attention). Bleakley et $a{ }^{\theta}{ }^{10}$ describe a typical MPTB at the start of an OT list as consisting of a technically-oriented discussion of the list led by the surgeon, equipment checks, patient lists and potential problems. In another study, team members first introduced themselves and their roles, develop a plan for the day and discuss critical aspects of each procedure as an MPTB. ${ }^{11}$ Bethune $e t ~ a l^{12}$ describe MPTB as including feedback from previous lists, consideration of external factors and a discussion of each patient on the list. Specific to our research, staff working in the hospitals involved in this study were encouraged to cover five steps in MPTB: (1) staff introduction including name and team role, (2) overview of the surgical list (eg, half-day/ full day, number of patients), (3) relevant details of each case, (4) questions and (5) summary of any changes or issues discussed. ${ }^{6}$

MPTB have been recognised as having positive effects on theatre outcomes. Studies show that they benefit teamwork ${ }^{7-14}$ safety, ${ }^{79-11} 13$ as well as efficiency. ${ }^{712}$ More generally, lack of standard methods for preoperative team communication has been identified, ${ }^{1}$ and MPTB are ideally placed to address this gap. Yet, they still remain an infrequent and underused practice in Australian OTs. ${ }^{213}$ Issues with uptake, or even resistance, are reported for other teamwork interventions in OTs (ie, checklists ${ }^{15-19}$ ). These include resistance from professionals in OTs, ${ }^{15-17}$ leadership and established hierarchies, ${ }^{17} 2021$ lack of education ${ }^{18}$ and poor communication. ${ }^{17} 19$

Given their relevance to teamwork and surgical outcomes, it is important to understand what is hindering and helping the uptake of MPTB in OTs. Grol and Grimshaw $^{22}$ identified that changes in clinical practice are only partially within the control of medical staff. There is a wider recognition in implementation research that implementation strategies need to be carefully targeted towards barriers or obstacles. ${ }^{22}{ }^{23}$ Accordingly, this study investigates the perceived barriers to the introduction of MPTB and solutions for overcoming these barriers. In recognition of the need for a systems approach towards change implementation and integration to work in $\mathrm{OTs}^{24}$ and healthcare more widely (see process normalisation theory, ${ }^{25}$ ) we apply Parker $e t a l$ s six-level model of work design influences ${ }^{26}$ to categorise the perceived barriers and solutions identified by surgical professionals. Parker et $a l^{26}$ identify six multilevel influences that interact with, and shape each other to affect work design in a particular situation. The six levels of influence on how work is organised within any particular situation include: global factors (eg, WHO strategies, global migration patterns that affect staffing), national factors (eg, Gross Domestic Product (GDP) and other economic aspects, government healthcare policies, industrial relations policies), occupational factors (eg, professional norms), organisational factors (eg, organisational design and culture), work group factors (eg, composition, local leadership) and individual factors (eg, education, motivation). To change work design, implementing MPTB in this case, or at least sustain a change in work design, one needs to adopt a full systems perspective to understand how multiple levels of influence shape work design and staff behaviours and how they interact with each other. Doing so recognises the complex nature of implementing quality improvement initiatives and can support individuals and organisations in their efforts to incorporate MPTB into standard practice.

\section{METHODS}

OT staff from four tertiary metropolitan hospitals were surveyed on their perceptions of perceived barriers towards conducting MPTB and the solutions for overcoming these perceived barriers. MPTB were encouraged at all sites; however, they were not fully integrated into daily practice. As part of presentations about MPTB at staff meetings, participants were asked to note down challenges they experienced in conducting MPTB in their workplaces, and to propose potential solutions to these challenges. A second wave of data collection was conducted via online surveys specifically addressed at surgeons and OT administrators as previous attempts to engage these groups were unsuccessful. Responses were provided in an open-ended text format and participants could provide as many or as few barriers and solutions as they wish (see online supplementary file provided). Participation was voluntary and anonymous. Participants were informed of the ways in which their responses would be used.

\section{Sample}

A sample of $n=103$ OT staff (out of which 44 were nurses, 13 technicians, 16 anaesthetists; 20 surgeons; 4 administrators; note that the remainder seven of the sample did not indicate their professional group) participated in this study. The data were collected over a 3-year period (wave 1; 2014-2017) and all OT staff were invited to contribute to this work on several occasions. Almost all nurses and anaesthetists attended information and training sessions 
where the opportunity to participate was given. Information and training sessions were not attended by surgeons and thus more direct methods of recruitment were necessary (eg, direct contact and surgical meeting attendance; wave 2,2019 ). The sample was of sufficient size for saturation to occur in our analysis. ${ }^{27}$

\section{Patient and public involvement}

No patients or members of the public were involved in this study.

\section{Data analysis}

Responses were coded independently by two raters following an inductive coding framework. ${ }^{28}$ Both raters were trained psychologists with a background in industrial and organisational psychology. The analysis was conducted in three steps. In the first step, one rater read all responses and identified emergent themes. In the second step, each response was assigned to one of the themes that had emerged during the first step. This second step involved two raters independently analysing the responses. Inter-rater reliability was assessed using Krippendorff's alpha and indicated that the raters were highly consistent in their coding of responses $\left(\alpha_{\text {Kripp }}=0.87\right.$, $\mathrm{CI}_{95 \% \mathrm{LL}} 0.80, \mathrm{CI}_{95 \% \mathrm{UL}} 0.94^{29}$ ). Results were generated by frequency counts per emergent theme, which is an indication of perceived relevance. ${ }^{30}$ In the final step, the identified themes were classified into the levels of work design influences by Parker et al. ${ }^{26}$

\section{RESULTS}

\section{Perceived barriers to the implementation of MPTB in OTs}

A total of 214 perceived barriers to implementing МРТВ in OTs were identified $(\mathrm{Md}=2$ per participant). These barriers reflected six themes. Table 1 shows the frequency of the themes identified in the perceived barriers and categorises these by systems levels. Notably barriers were predominantly identified at the work group level, one of the lowest systems levels described by Parker et al ${ }^{26}$ $(\mathrm{f}=132)$. Within the work group level, the majority of responses ( $\mathrm{f}=117$ ) focused on staff not being in the OT at the same time at the start of a list and conflicting tasks as a key barrier to MPTB, making this issue the most common barrier to holding briefings. This barrier was sometimes attributed to various reasons such as having different start times, setups or staff being late. Further, communication issues, such as confusion due to information accuracy and specificity (eg, around procedure details, equipment needs), or challenges in interacting constructively with other team members were also reported as a barrier at the work group level $(\mathrm{f}=15)$. Next most frequently, perceived barriers were reported at the individual level $(\mathrm{f}=48)$.

Table 1 Illustrative quotes and frequencies of each barrier

\begin{tabular}{|c|c|c|c|}
\hline Theme & Example quotes & $\begin{array}{l}\text { Frequency } \\
(f)\end{array}$ & $\begin{array}{l}\text { Frequency } \\
\text { (\% of total)* }^{*}\end{array}$ \\
\hline Organisational-level barriers & & 34 & 15.81 \\
\hline Organisational constraints & $\begin{array}{l}\text { Organisations audit the start time/briefings not accommodated in } \\
\text { schedule } \\
\text { Inadequate staffing } \\
\text { AM list overruns, affecting PM list start time }\end{array}$ & 14 & 6.51 \\
\hline Work group-level barriers & & 132 & 61.39 \\
\hline $\begin{array}{l}\text { Not everyone present/ } \\
\text { conflicting tasks }\end{array}$ & $\begin{array}{l}\text { Surgeon or anaesthetists finishing rounds } \\
\text { Complex set ups } \\
\text { Staff nurses busy locating equipment } \\
\text { Late team members } \\
\text { Team members are not available before or at } 8 \text { am }\end{array}$ & 117 & 54.42 \\
\hline Individual-level barriers & & 48 & 22.33 \\
\hline Negative attitudes & $\begin{array}{l}\text { Briefings [are] done as a formality with steps missing and no space for } \\
\text { questions } \\
\text { Not interested, refuses to participate } \\
\text { Not supportive of the process }\end{array}$ & 38 & 17.67 \\
\hline Lack of knowledge & $\begin{array}{l}\text { Visiting surgeons not knowing procedures } \\
\text { Staff present during the briefing may be different to ones involved in } \\
\text { specific surgeries } \\
\text { Junior staff not being aware of briefings }\end{array}$ & 10 & 4.65 \\
\hline
\end{tabular}

${ }^{*}$ Total of 214 responses. 
Table 2 Illustrative quotes and frequencies of each solution

\begin{tabular}{|c|c|c|c|}
\hline Theme & Example quotes & $\begin{array}{l}\text { Frequency } \\
(f)\end{array}$ & $\begin{array}{l}\text { Frequency } \\
\text { (\% of total)* }\end{array}$ \\
\hline Organisational-level solutions & & 103 & 74.10 \\
\hline Organisational policy changes & $\begin{array}{l}\text { Making it a hospital policy/audit to ensure all members are present } \\
\text { Making it mandatory }\end{array}$ & 13 & 9.35 \\
\hline Culture change & $\begin{array}{l}\text { Encouragement, senior support, management support } \\
\text { Need engaged anaesthetic lead }\end{array}$ & 14 & 10.07 \\
\hline Better communication & $\begin{array}{l}\text { Communicate } \\
\text { Having team leaders who are good communicators }\end{array}$ & 21 & 15.12 \\
\hline Enforcing briefings as a priority & $\begin{array}{l}\text { Make it a priority } \\
\text { Do not bring patient into the OR until briefing is done } \\
\text { Preparing beforehand }\end{array}$ & 15 & 10.79 \\
\hline
\end{tabular}

${ }^{*}$ Total of 139 responses.

These perceived barriers include lack of knowledge about MPTB $(\mathrm{f}=10)$ and negative attitudes towards MPTB $(\mathrm{f}=38)$. Such negative attitudes included staff not taking briefings serious and not seeing the benefit of them, as well as active resistance to briefings. Two perceived barriers at the organisational level were reported $(\mathrm{f}=34)$, namely surgical list attributes $(\mathrm{f}=20$; eg, lists with only emergency cases, variation in surgical staff) and organisational constraints ( $\mathrm{f}=14$; eg, previous list runs over, inadequate staffing). Ccomparisons of frequencies of barriers reported by surgeons and nurses showed identical rankings of the three levels (ie, work group barriers were most frequently identified in the two professional groups, followed by individual and organisational barriers).

\section{Solutions to MPTB implementations}

A total of 139 potential solutions for overcoming perceived barriers to implementing MPTB were provided by the participants $(\mathrm{Md}=1$ solution per participant). Within the suggested solutions, a total of seven themes were identified. Table 2 illustrates the frequency and content of each solution. Solutions resided at organisational $(\mathrm{f}=103)$ and work group level $(\mathrm{f}=36)$. At organisational level, participants suggested changes to staffing and scheduling $(\mathrm{f}=39)$ and education around the benefits and procedures for effective MPTB as solutions to MPTB implementation $(\mathrm{f}=34)$. A number of solutions at organisational level emerged with relatively low frequency. Participants suggested instituting organisational changes via policies ( $\mathrm{f}=13$; eg, making briefings mandatory) and organisational culture change $(\mathrm{f}=14)$. Further, use of technology, such as phones, was also mentioned $(\mathrm{f}=3)$.

At work group level, two solutions emerged, namely better communication within the OT team to ensure high-quality briefings $(\mathrm{f}=21)$ and strategies that allow team members to enforce briefings as a priority within each team $(\mathrm{f}=15)$.

\section{DISCUSSION}

This qualitative study identifies perceived barriers to the implementation of MPTB in OTs and potential solutions for overcoming these perceived barriers. Investigating what hinders such briefings from occurring is important as MPTB can support effective teamwork and communication in OT teams. Previous research has shown that similar quality improvements have faced resistance in $\mathrm{OTs}^{4}$ and emphasises a systems approach towards team interventions. ${ }^{24}$ To assist operating staff in implementing MPTB as a day-to-day practice, it is important to identify and understand potential barriers that may make the implementation of MPTB more difficult so that solutions can be targeted towards overcoming these specific issues. The present research identifies the barriers and solutions specific to MPTB implementation. In doing so, it can assist practitioners and hospital administrators wanting to implement MPTB into their standard practice. The present study focuses uniquely on the perceived barriers to MPTB implementation as previous research into the implementation of healthcare interventions has 
highlighted the necessity to conduct a thorough barrier analysis prior to trying to implement a new practice as these barriers can often result in an intervention failing regardless of the facilitating factors. ${ }^{23} 31$ Our findings extend this approach by not only identifying the barriers themselves but also soliciting insights from staff as to the potential solutions. By identifying potential solutions to perceived barriers to implementation, hospitals seeking to implement MPTB will be better equipped to proactively manage potential barriers ${ }^{32}$ and can design comprehensive and targeted strategies to address barriers to change. ${ }^{22}$

Barriers to MPTB identified in this study were at organisational, work group and individual levels, with the majority occurring at work group level. A number of these barriers (eg, issues related to attitudes and knowledge) identified in this study are similar to those that have been identified in relation to the implementation of the WHO checklists. ${ }^{4}$ The focus at work group level reflects MPTB's status as a team-level work design intervention $^{6}$; however, the emergence of barriers at other levels illustrates the relevance of a systems view on MPTB implementation. Notably, no barriers at the occupational, national or global levels were identified by respondents in this study. Despite this finding, we posit, based on Parker $e t a l \mathrm{~s}^{26}$ systems model of work design influences and previous research on change implementation in medical settings $^{322}$ that such barriers exist, but that OT staff were not necessarily aware of them. In contrast to immediate barriers at the individual and work group levels that are likely salient in the day-to-day experience of OT staff, barriers existing at the occupational, national and global levels likely shape MPTB implementation indirectly in more subtle ways that are often difficult to identify. Crucially though, these barriers at occupational, national or global level are wide reaching and the successful adoption of strategies designed to overcome these challenges are likely essential for sustained change. ${ }^{22} 24{ }^{26}$ In the case of MPTB, at occupational level, barriers such as surgical work practices that apply across multiple hospitals (such as the consultancy model of surgeon work), and at national-level issues such as healthcare funding models are likely to be relevant.

Consolidating our results and the above discussion into a process model of systems barriers to MPTB occurrence $\left(\right.$ based $\left.o^{26}\right)$, figure 1 illustrates the complex interactions of barriers at various systems levels identified in this study and how they can contribute to MPTB occurrence. We identify staff not being in the room and being occupied with conflicting tasks as a core, immediate bottleneck to MPTB occurrence from our findings (based on it being the most frequently identified barrier).

The remaining barriers are likely to affect staff presence for MPTB attendance and to interact with each other. For example, at the individual level, attitudes are likely to be directly linked to staff presence for MPTB but are also likely to adversely affect communication, which in turn can also contribute to staff not being present for MPTB. Further, attitudes are also likely to be shaped by organisational constraints, such as inadequate staffing levels, which can contribute to staff dismissing MPTB, as it can appear like another task they need to engage in.

Participants generated fewer solutions for MPTB implementation than barriers. However, the content of their responses was varied, so that a wide range of solutions could be identified. Solutions for MPTB implementation that participants generated resided at the organisational and work group levels only. Similar to the barriers to MPTB, participants did not report higher system levels solutions at either the occupational or national levels. As has been argued above, barriers and solutions are likely to exit at each level. The criticality of higher level solutions becomes clear when matching barriers and solutions based on their content (see content in grey in figure 1). Content matching illustrates that solutions are likely to address barriers that reside at the same system level or
Solutions

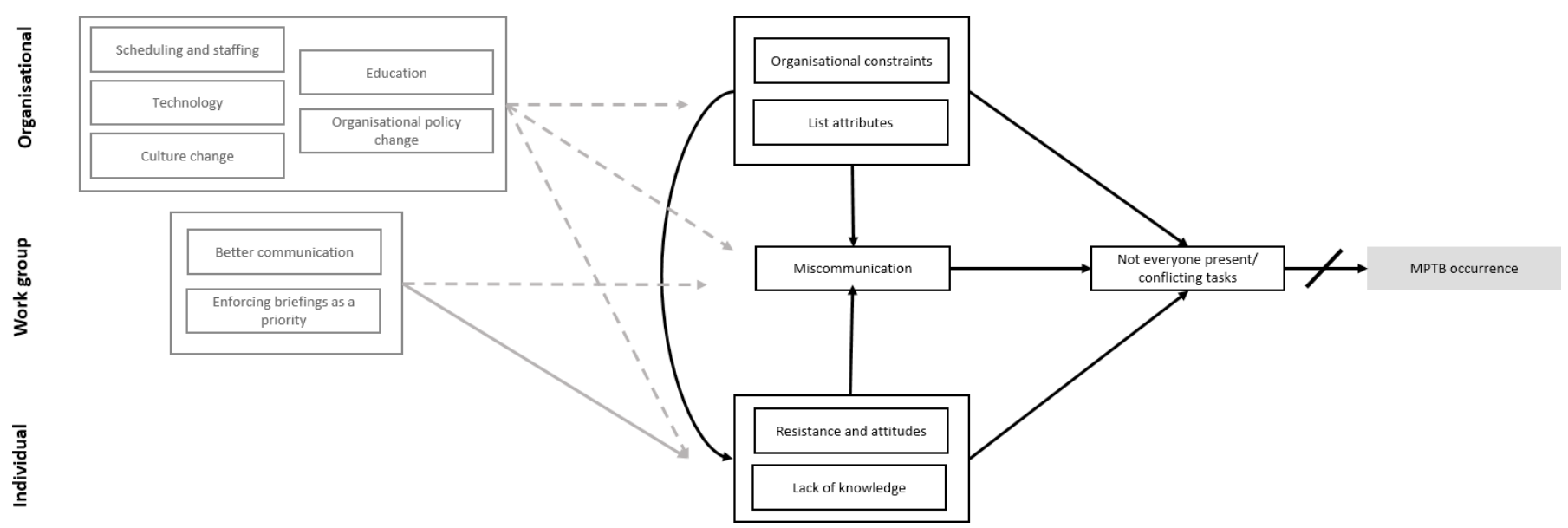

Figure 1 A process model of work design barriers to MPTB occurrence. MPTB, multiprofessional team briefings.

\section{Barriers}


below. However, a solution is unlikely to reach a barrier at a level that exceeds the level of the solution. As is shown in figure 1, solutions at the organisational level are likely to also address barriers at the same level or below, but not above. For example, scheduling and staffing solutions can address organisational constraints (ie, organisationallevel barrier), as well as presence of staff at MPTB (work group-level barrier), thereby addressing barriers at organisational and team levels. However, they are unlikely to reach barriers at occupational or national levels. Similarly, education of OT staff can address the individuallevel barrier of attitudes by clarifying the benefits of team briefings, and reduce miscommunication at the team level. However, education of OT staff is unlikely to address barriers that reside above the organisational level, such as cost minimisation or staffing levels. While education emerged in our study as an individual-level solution with a focus on OT staff, it needs to be recognised that differently targeted education can also facilitate MPBT implementation at higher system levels. To address barriers at higher levels, concerted efforts can be taken to actively disseminate research findings and educate policy-makers so that best practices can be fully endorsed and adopted by health departments and included in their standards of patient care.

Future research may consider investigating the processes involved in MPTB implementation via case studies and in-depths interviews with OT staff and administrators. Such case studies may identify more detailed information on the barriers and solutions for MPTB implementation identified here. Crucially, our study identifies perceived barriers, however, it is unclear to what extent these overlap with actual barriers to MPTB implementation. Further, longitudinal investigations of barriers and solutions over the course of MPTB implementation may help illuminate the dynamic relationship between barriers and solutions at different levels and provide a process perspective to this type of quality improvement initiatives.

It also needs to be considered that strategies that do not directly target MPTB implementation may also have benefits for their wider implementation success. As many other strategies around communication in healthcare, MPTB are rooted in established practices of crew resource management (CRM) in aviation and other industries. ${ }^{13} 3334$ In addition to the solutions identified in this study, the implementation of MPTB in practice may also benefit from considering other practices from CRM team training targeted at teamwork and communication by growing awareness and appreciation for teamwork efforts like MPTB more generally.

\section{Study strengths and weaknesses}

This is the first study to investigate the perceived barriers and solutions to the implementation of MPTB in OTs. The research adopts a multilevel systems approach grounded in theory, which has generated practical guidance and solutions and illustrates the complexity of MPTB implementation. The data were collected via anonymised open-ended survey questions. Using surveys, rather than interviews, allowed for inclusions of a larger group of participants, so that the results are more representative. Responses provided a high level summary that captured the issues well, however, did not generate more in depth reflection as to why participants perceive specific barriers and potential solutions. Further, Grol and Grimshaw $^{22}$ describe barriers and facilitators of change in clinical work contexts. However, our study only focused on barriers and examined the strategies to overcome them (ie, solutions), so that other aspects that may need to be considered for successful implementation were not captured. In particular, our study, while addressing one of the key issues associated with implementation processes did not consider facilitators, as issues that may suport the implementation of MPTB in OTs. ${ }^{22}$ Such facilitators of change implementations in clinical contexts may include incentives, feedback or perceived social norms. ${ }^{22}$ Finally, our study captured the frequency with which barriers and solutions were reported. It needs to be noted that, while frequency in content analysis has been described as a marker of relevance,${ }^{30}$ it may be affected by awareness, or other factors that may lead participants to refer to one issue over another.

\section{Conclusion and implications}

Considering the barriers and solutions to MPTB implementation, this paper illustrates that a work design change needs to be built on an understanding of how multiple systems levels shape work designs and behaviours in OTs. Barriers and solutions to MPTB' implementation were predominantly reported by OT staff at work group level, whereas solutions were most likely to reside at organisational level. Notably, our participants did not identify higher level barriers and solutions at occupational or national levels. Yet, our matching of barriers and solutions to MPTB implementation illustrates the possible limitations of lower level solutions in overcoming wider systemic changes in the underlying processes that are necessary to sustain the implementation of MPTB. Our findings reinforce the importance of systems-based change in generating adequate ways of addressing common barriers to MPTB implementation.

\section{Author affiliations}

${ }^{1}$ School of Psychological Science, The University of Western Australia, Crawley, Western Australia, Australia

${ }^{2}$ Business School, The University of Western Australia, Crawley, Western Australia, Australia

${ }^{3}$ Future of Work Institute, Curtin University, Perth, Western Australia, Australia ${ }^{4}$ Division of Obstetrics and Gynaecology, Faculty of Health and Medical Sciences, The University of Western Australia, Crawley, Western Australia, Australia ${ }^{5}$ Faculty of Health and Medical Sciences, Surgery, The University of Western Australia, Crawley, Western Australia, Australia

Acknowledgements We thank Fiona Stanley Hospital and Fremantle Hospital, as well as two other hospitals in WA for collaborating with us. We in particular want to express our gratitude to the medical, nursing, administrative and technical staff in the operating theatres who volunteered their valuable time and insights. 
Contributors SKP was the principal investigator for this study. LF and JAC distributed the surveys. LF analysed the responses (together with a research assistant) on which the paper is based and led on writing this manuscript. LF, SKP, JAC, AFSF, and YL contributed to the design of the study and editing of the final manuscript and the refining of its intellectual content.

Funding This work was supported by the Government of Western Australia Department of Health grant number (F-AA-33992).

Competing interests None declared.

Patient consent for publication Not required.

Ethics approval This research was granted ethical approval by the Human Research Ethics Office at the University of Western Australia (approval number/ID: $\mathrm{RA} / 4 / 1 / 6933$ ) and was performed in accordance with ethical standards.

Provenance and peer review Not commissioned; externally peer reviewed.

Data availability statement No data are available. The datasets analysed for the current study are not publicly available, as participants were not asked to consent to the sharing of the data at the time of data collection.

Open access This is an open access article distributed in accordance with the Creative Commons Attribution Non Commercial (CC BY-NC 4.0) license, which permits others to distribute, remix, adapt, build upon this work non-commercially, and license their derivative works on different terms, provided the original work is properly cited, appropriate credit is given, any changes made indicated, and the use is non-commercial. See: http://creativecommons.org/licenses/by-nc/4.0/.

\section{ORCID iDs}

Laura Fruhen http://orcid.org/0000-0002-9599-3468

Joseph Alexandre Carpini http://orcid.org/0000-0002-4694-2672

\section{REFERENCES}

1 Cruz SA, Idowu O, Ho A, et al. Differing perceptions of preoperative communication among surgical team members. Am J Surg 2019;217:1-6.

2 Fruhen LS, Carpini J, Parker SK. Let's Talk Teamwork: Multiprofessional team briefings in WA operating theatres. Surgical News, The Royal Australian College of Surgeons 2017;18:16-18.

3 Abbott TEF, Ahmad T, Phull MK, et al. The surgical safety checklist and patient outcomes after surgery: a prospective observational cohort study, systematic review and meta-analysis. $\mathrm{Br} J$ Anaesth 2018;120:146-55.

4 Treadwell JR, Lucas S, Tsou AY. Surgical checklists: a systematic review of impacts and implementation. BMJ Qual Saf 2014;23:299-318.

5 International Surgical Outcomes Study group. Global patient outcomes after elective surgery: prospective cohort study in 27 low-, middle- and high-income countries. Br J Anaesth 2016;117:601-9.

6 Carpini JA, Flemming AFS, Parker SK. Multidisciplinary team briefings: a way forward. Day Surgery Australia 2015;14:12-14.

7 Leong KBMSL, Hanskamp-Sebregts M, van der Wal RA, et al. Effects of perioperative briefing and Debriefing on patient safety: a prospective intervention study. BMJ Open 2017;7:e018367.

8 NHS Improvement. Provisional publication of never events reported as occurring between 1 April and 31 October. London: NHS Improvement, 2018

9 Bleakley A, Allard J, Hobbs A. Towards culture change in the operating theatre: embedding a complex educational intervention to improve teamwork climate. Med Teach 2012;34:e635-40.

10 Bleakley A, Boyden J, Hobbs A, et al. Improving teamwork climate in operating theatres:The shift from multiprofessionalismto interprofessionalism. J Interprof Care 2006;20:461-70.
11 Khoshbin A, Lingard L, Wright JG. Evaluation of preoperative and perioperative operating room briefings at the hospital for sick children. Can J Surg 2009;52:309-15.

12 Bethune R, Sasirekha G, Sahu A, et al. Use of briefings and debriefings as a tool in improving team work, efficiency, and communication in the operating theatre. Postgrad Med $\mathrm{J}$ 2011;87:331-4

13 Allard J, Bleakley A, Hobbs A, et al. "Who's on the team today?" The status of briefing amongst operating theatre practitioners in one UK hospital. J Interprof Care 2007;21:189-206.

14 Allard J, Bleakley A, Hobbs A, et al. Pre-surgery briefings and safety climate in the operating theatre. BMJ Qual Saf 2011;20:711-7.

15 Sparkes D, Rylah B. The world Health organization surgical safety checklist. Br J Hosp Med 2010;71:276-80.

16 Sewell M, Adebibe M, Jayakumar P, et al. Use of the who surgical safety checklist in trauma and orthopaedic patients. Int Orthop 2011;35:897-901.

17 Conley DM, Singer SJ, Edmondson L, et al. Effective surgical safety checklist implementation. J Am Coll Surg 2011;212:873-9.

18 Levy SM, Senter CE, Hawkins RB, et al. Implementing a surgical checklist: more than checking a box. Surgery 2012;152:331-6.

19 Fourcade A, Blache J-L, Grenier C, et al. Barriers to staff adoption of a surgical safety checklist. BMJ Qual Saf 2012;21:191-7.

20 Helmiö P, Takala A, Aaltonen L-M, et al. First year with who surgical safety checklist in 7148 otorhinolaryngological operations: use and user attitudes. Clin Otolaryngol 2012;37:305-8.

21 Yuan CT, Walsh D, Tomarken JL, et al. Incorporating the world Health organization surgical safety checklist into practice at two hospitals in Liberia. Jt Comm J Qual Patient Saf 2012;38:254-60.

22 Grol R, Grimshaw J. From best evidence to best practice: effective implementation of change in patients' care. The Lancet 2003;362:1225-30.

23 Bauer MS, Damschroder L, Hagedorn H, et al. An introduction to implementation science for the non-specialist. BMC Psychology 2015;3:32.

24 Catchpole K, Russ S. The problem with checklists. BMJ Qual Saf 2015;24:545-9.

25 May C. Towards a general theory of implementation. Implement Sci 2013;8:18.

26 Parker SK, Van den Broeck A, Holman D. Work design influences: a synthesis of multilevel factors that affect the design of jobs. Acad Manag Ann 2017;11:267-308.

27 O’Reilly M, Parker N. 'Unsatisfactory Saturation': a critical exploration of the notion of saturated sample sizes in qualitative research. Qualitative Research 2013;13:190-7.

28 Gioia DA, Corley KG, Hamilton AL. Seeking Qualitative Rigor in Inductive Research:Notes on the Gioia Methodology. Organizational Research Methods 2013;16:15-31.

29 Hayes AF, Krippendorff K. Answering the call for a standard reliability measure for coding data. Commun Methods Meas 2007;1:77-89.

30 Namey E, Guest G, Thairu L. Data reduction techniques for large qualitative data sets. In: GGaK M, ed. Handbook for team-based qualitative research. Plymouth, UK: AltaMira Press, 2008: 137-59.

31 Shojania KG, Grimshaw JM. Evidence-Based quality improvement: the state of the science. Health Aff 2005;24:138-50.

32 Bosch $\mathrm{M}$, van der Weijden $\mathrm{T}$, Wensing $\mathrm{M}$, et al. Tailoring quality improvement interventions to identified barriers: a multiple case analysis. J Eval Clin Pract 2007;13:161-8.

33 O'Connor P, Flin R. Crew resource management training for offshore oil production teams. Saf Sci 2003;41:591-609.

34 Salas E, Burke CS, Bowers CA, et al. Team training in the skies: does Crew resource management (CRM) training work? Hum Factors 2001;43:641-74. 